Virginia Commonwealth University VCU Scholars Compass

\title{
Electrically and magnetically tunable phase shifters based on a barium strontium titanate-yttrium iron garnet layered structure
}

\author{
J.H. Leach \\ Virginia Commonwealth University, s2jleach@vcu.edu \\ H. Liu \\ Virginia Commonwealth University \\ V.Avrutin \\ Virginia Commonwealth University, vavrutin@vcu.edu \\ See next page for additional authors
}

Follow this and additional works at: http://scholarscompass.vcu.edu/egre_pubs

Part of the Electrical and Computer Engineering Commons

Leach, J. H., Liu, H., \& Avrutin, V., et al. Electrically and magnetically tunable phase shifters based on a barium strontium titanate-yttrium iron garnet layered structure. Journal of Applied Physics, 108, 064106 (2010). Copyright (C) 2010

American Institute of Physics.

\section{Downloaded from}

http://scholarscompass.vcu.edu/egre_pubs/156

\footnotetext{
This Article is brought to you for free and open access by the Dept. of Electrical and Computer Engineering at VCU Scholars Compass. It has been accepted for inclusion in Electrical and Computer Engineering Publications by an authorized administrator of VCU Scholars Compass. For more information, please contact libcompass@vcu.edu.
} 
Authors

J. H. Leach, H. Liu, V. Avrutin, E. Rowe, Ü. Özgür, H. Morkoç, Y.-Y. Song, and M. Wu 


\title{
Electrically and magnetically tunable phase shifters based on a barium strontium titanate-yttrium iron garnet layered structure
}

\author{
J. H. Leach, ${ }^{1, a)}$ H. Liu, ${ }^{1}$ V. Avrutin, ${ }^{1}$ E. Rowe, ${ }^{1}$ Ü. Özgür, ${ }^{1}$ H. Morkoç, ${ }^{1}$ Y.-Y. Song, ${ }^{2}$ and \\ M. $\mathrm{Wu}^{2}$ \\ ${ }^{1}$ Department of Electrical and Computer Engineering, Virginia Commonwealth University, Richmond, \\ Virginia 23284, USA \\ ${ }^{2}$ Department of Physics, Colorado State University, Fort Collins, Colorado 80523, USA
}

(Received 15 March 2010; accepted 15 July 2010; published online 20 September 2010)

\begin{abstract}
We report on the tuning of permittivity and permeability of a ferroelectric/ferromagnetic bilayer structure which can be used as a microwave phase shifter with two degrees of tuning freedom. The structure was prepared by the growth of a yttrium iron garnet (YIG) layer on a gadolinium gallium garnet substrate by liquid phase epitaxy, the growth of a barium strontium titanate (BST) layer on the YIG layer through pulsed laser deposition, and then the fabrication of a coplanar waveguide on the top of BST through e-beam evaporation and trilayer liftoff techniques. The phase shifters exhibit a differential phase shift of $38 \% \mathrm{~cm}$ at $6 \mathrm{GHz}$ through permittivity tuning under an applied electric field of $\sim 75 \mathrm{kV} / \mathrm{cm}$ and a static magnetic field of 1700 Oe. By tuning the permeability through the applied magnetic field we increase the differential phase shift to $52 \% \mathrm{~cm}$ and simultaneously obtain a better match to the zero applied electric field condition, resulting in an improvement in the return loss from 22.4 to $24.9 \mathrm{~dB}$. Additionally, we demonstrate the use of a lead magnesium niobate-lead titanate (PMN-PT) layer to tune the permeability of the YIG layer. This tuning relies on the piezoelectric and magnetostrictive effects of PMN-PT and YIG, respectively. Tuning of the ferromagnetic response through strain and magnetostriction as opposed to applied magnetic field can potentially pave the way for low power consumption, continuously and rapidly tunable, impedance matched phase shifters. (C) 2010 American Institute of Physics. [doi:10.1063/1.3486463]
\end{abstract}

\section{INTRODUCTION}

The ability to rapidly change the dielectric constant of a ferroelectric material under the application of a dc electric field allows one to design tunable microwave components with low cost and compatibility with thin film technologies. ${ }^{1}$ However, with such an approach to tunable microwave devices, particularly for phase shifters and antennas, there is a change in the impedance of the device associated with the aforementioned change in dielectric constant. ${ }^{2}$ This gives rise to impedance mismatches, and hence, losses in the system. That said, by incorporating a ferrite material into a device, one gains the ability to change both the dielectric constant, $\varepsilon$, and the permeability, $\mu$, allowing one to control the impedance, $\eta \propto \sqrt{\mu / \varepsilon}$ independently of the phase shift (through $\varepsilon$ or $\mu){ }^{3}$ In fact, the integration of ferrites with ferroelectric materials is not a new concept and pervious efforts have already resulted in a new class of tunable microwave devices based on the propagation of hybrid spin electromagnetic waves. ${ }^{4,5}$ Although very successful in resonator schemes, and boasting impressive differential phase shifts, phase shifters based on the spin wave phenomenon have high insertion losses, particularly outside of a narrow frequency range in which they are designed to operate. Alternatively, ferroelectric/ferrite bilayers have been used for phase shifters operating near the ferromagnetic resonance (FMR) frequency of the ferrite, with a piezoelectric stressor employed to shift the FMR frequency, in turn inducing a differential phase

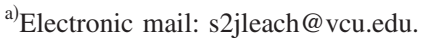

shift. ${ }^{6}$ However, without independently controlling $\varepsilon$ and $\mu$ in a medium, a phase shifter will have changing characteristic impedance as it changes its phase. In this work, we employ a $\mathrm{Ba}_{0.5} \mathrm{Sr}_{0.5} \mathrm{TiO}_{3}(\mathrm{BST})-\mathrm{Y}_{3} \mathrm{Fe}_{5} \mathrm{O}_{12}$ (YIG) bilayer on a gadolinium gallium garnet (GGG) substrate and use a coplanar waveguide geometry, in order to demonstrate the ability to independently tune the permittivity and permeability of the overall device, resulting in phase shifts at constant characteristic impedances. The permittivity of the BST component is tunable with an electric field applied across the coplanar waveguide (CPW) signal/ground while the permeability of the YIG component is tunable with a biasing magnetic field along the signal. Additionally, after bonding a piezoelectric lead magnesium niobate-lead titanate (PMNPT) layer to the backside of the structure after removing the GGG substrate, we were able to tune the permeability of the YIG by applying an electric field to the PMN-PT, as opposed to changing the bias magnetic field. The mechanical deformation of the PMN-PT is transferred to the YIG, causing a shift in the FMR frequency of the YIG. ${ }^{7,8}$ Such a structure is desirable since electric field tuning of the ferrite material through magnetostriction constitutes a much more efficient and rapid way of tuning as opposed to changing the biasing magnetic field.

\section{EXPERIMENT}

The BST-YIG bilayer structure shown in Fig. 1 was prepared by a two step process: (1) the growth of a YIG layer on a GGG substrate by liquid phase epitaxy and (2) the deposi- 

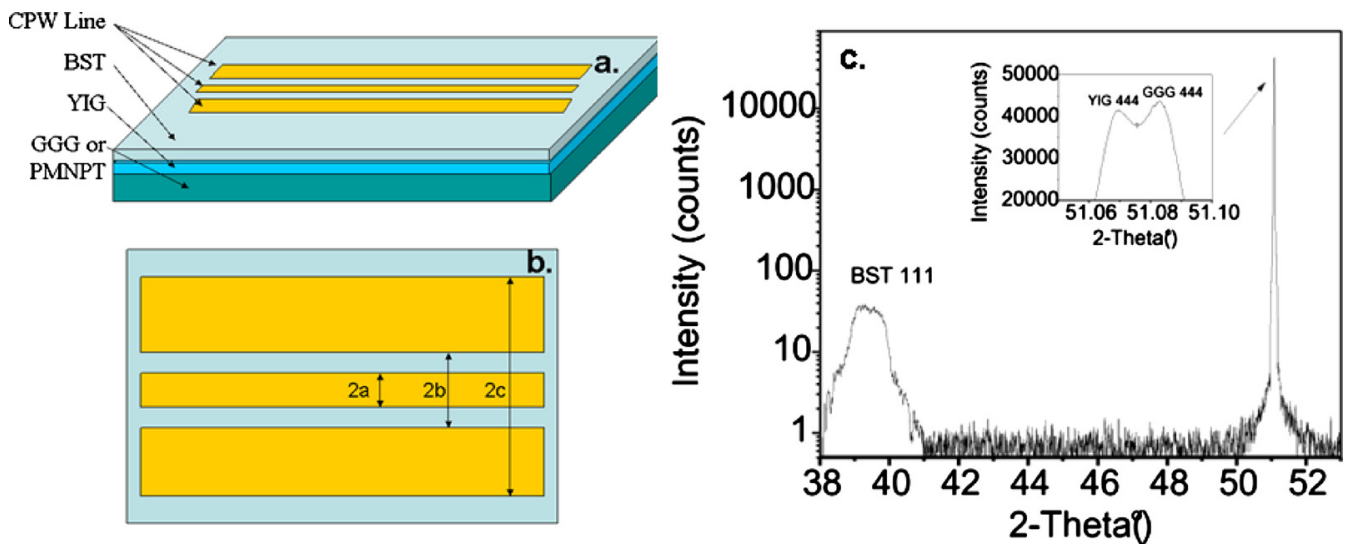

FIG. 1. (Color online) (a) Schematic of the BST/YIG/GGG or BST/YIG/PMN-PT bilayer phase shifter structure. (b) Top view of the CPW geometry with the line widths of interest indicated. (c) $2 \theta-\omega$ scan of the BST/YIG/GGG structure after growth.

tion of a BST layer on the YIG layer by pulsed laser deposition. The BST layer was deposited at $800{ }^{\circ} \mathrm{C}$ under oxygen atmosphere at $75 \mathrm{mTorr}$. The laser energy fluence and repetition rate were $1.7 \mathrm{~J} / \mathrm{cm}^{2}$ and $25 \mathrm{~Hz}$, respectively. The resulting thicknesses of the YIG and BST layers are about $5.3 \mu \mathrm{m}$ and $5.0 \mu \mathrm{m}$, respectively. Subsequently, the orientation and crystal quality of the epitaxial films was assessed using high resolution x-ray diffraction (HR-XRD).

Next, a set of CPW lines with lengths of 1.5, 1.1, 0.25, and $0.2 \mathrm{~mm}$ were fabricated on the surface of the BST-YIG structure using a trilayer liftoff procedure resulting in a metal stack of $\mathrm{Cr} / \mathrm{Ti} / \mathrm{Ag} / \mathrm{Au}(50 / 25 / 2000 / 50 \mathrm{~nm})$ deposited by electron beam evaporation (thermal evaporation for $\mathrm{Au}$ ). The $68 \mu \mathrm{m}$-wide center conductor line is placed between two $235 \mu \mathrm{m}$-wide ground plane conductors with a separation of $4 \mu \mathrm{m}$ as shown in Fig. 1.

In order to derive the dielectric and magnetic properties of the phase shifters, the thru-reflect-line (TRL) calibration method was used to determine the propagation parameters (the phase and loss constants) ${ }^{9,10}$ of the set of coplanar waveguide lines fabricated on the ferroelectric/ferrite bilayer. Then conformal mapping was used to extract the dielectric and magnetic properties of individual layers. ${ }^{11}$ The scattering parameters were measured using an HP8510B vector network analyzer and a set of CPW probes in the frequency range from 4 to $12 \mathrm{GHz}$. The calibration was performed using the on-wafer CPW lines once in absence of an external magnetic field and again under an applied magnetic field of 1700 Oe. The dc electric field was applied through the test set using the same CPW probes. For measurements under magnetic fields, the sample was placed on top of a set of permanent magnets so that the field was parallel to the direction of microwave energy propagation (along the CPW lines), and by changing the distance of the sample from the permanent magnets the field was varied from $\sim 1600$ to 1800 Oe.

After determining the dielectric and magnetic properties of the bilayer, we replaced most of the GGG substrate with a piezoelectric slab ( $\sim 300 \mu \mathrm{m}$ thick) of PMN-PT. This was achieved by mechanically polishing the GGG substrate until only a thin $(\sim 20 \mu \mathrm{m})$ portion of the GGG substrate remained. The bulk $\langle 111\rangle$ PMN-PT substrate with $\mathrm{Cr} / \mathrm{Au}$
$(50 / 50 \mathrm{~nm})$ electrodes on both sides was then mechanically bonded to the remaining BST/YIG/GGG structure using a cynoacrylate-based adhesive. Microwave measurements were again performed using different applied electric fields to the PMN-PT structure.

\section{RESULTS AND DISCUSSION}

\section{A. Dielectric and magnetic properties}

Structural quality and orientation of the YIG and BST films were determined using HR-XRD. $2 \theta-\omega$ curves [Fig. 1 (c)] confirmed the orientation of the both the YIG and BST films to be $\langle 111\rangle$. Furthermore, the full-width at half maximum (FWHM) of the $\omega$ rocking curve of the YIG film of 29 arc sec demonstrates the high crystalline quality of the YIG. However, the FWHM of the BST film could not be assessed due to broadening in $2 \theta$, presumably due to inferior crystal quality or a nonuniform $d$-spacing throughout the film.

Figure 2 shows the result from the TRL calibration, the square of the normalized effective phase constant (which is the imaginary part of the propagation constant, $\gamma$, normalized by c/ $\omega$ ), as well as the loss (in decibel per centimeter) for the bilayer structure in the absence of magnetic field (thin lines) and under an applied magnetic field of $\sim 1700$ Oe (thick lines). Measurements were performed without applied electric fields. Several results are evident from Fig. 2. First, in

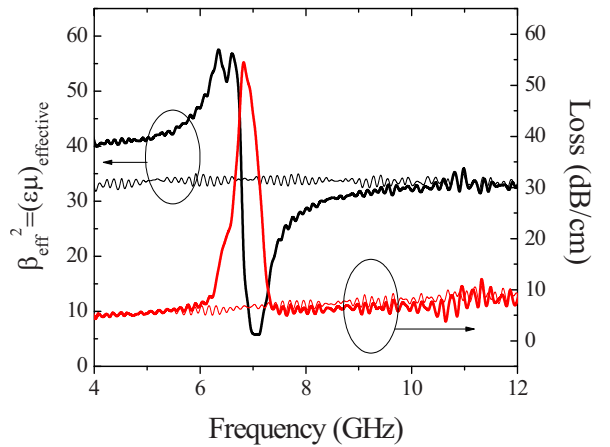

FIG. 2. (Color online) Square of the effective phase constant, $\beta_{\text {eff }}$, and loss (in $\mathrm{dB} / \mathrm{cm}$ ) for the BST/YIG bilayer in the absence of magnetic fields (thin lines) and under a dc magnetic field of 1700 Oe (thick lines). 

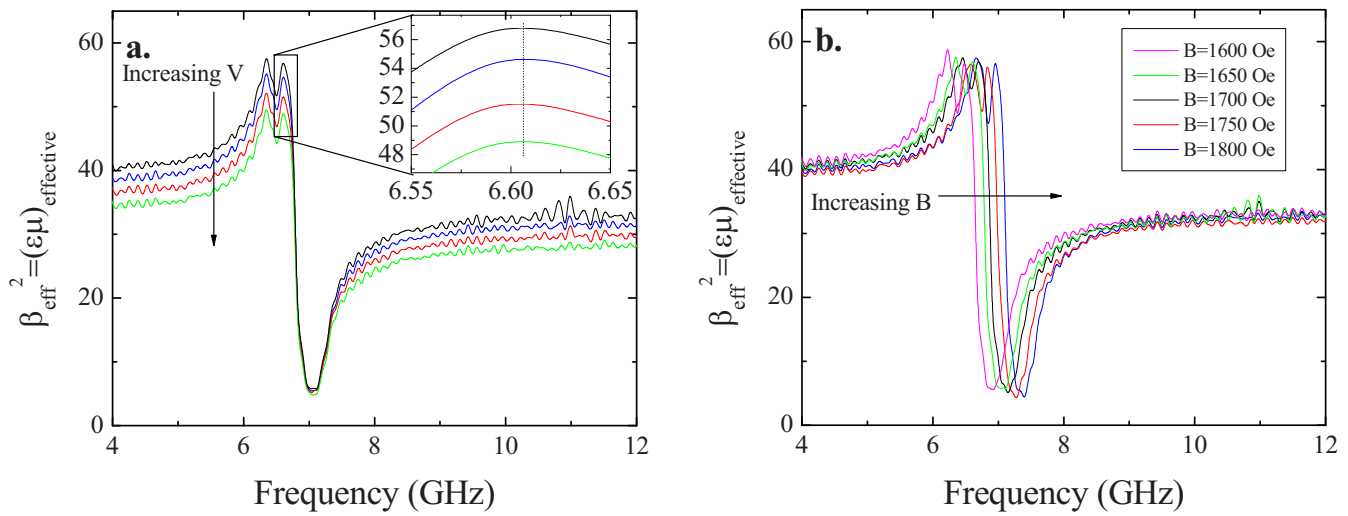

FIG. 3. (Color online) Tuning of the effective phase constant of the bilayer (a) by changing the applied electric field (applied voltage ranges from 0 to $30 \mathrm{~V}$ in steps of $10 \mathrm{~V}$ ) under static magnetic field and (b) by changing the applied magnetic field (applied field ranges from 1600 to 1800 Oe in steps of 50 Oe) under zero electric field.

the absence of magnetic fields, both phase and loss profiles are relatively constant over the entire frequency range, and the loss level is relatively high, about $5-10 \mathrm{~dB} / \mathrm{cm}$. This high value of loss is primarily due to the relatively low impedance of the CPW line $(\sim 11 \Omega)$, which results in large ohmic loss. ${ }^{10,11}$ Second, the application of an external magnetic field of 1700 Oe leads to a resonance behavior in the phase profile: an increased phase constant at low frequencies and a rapid change with frequency in the profile around $6.75 \mathrm{GHz}$. Third, the loss profile shows a peak near the frequency at which the phase constant changes rapidly, and the peak loss is about $55 \mathrm{~dB} / \mathrm{cm}$ which is well above the loss level for the no-field case. These results are attributed to the FMR in the YIG layer, and this is confirmed by the fact that the resonance frequency $6.75 \mathrm{GHz}$ is very close to the theoretical FMR frequency value $6.78 \mathrm{GHz}$. It is this resonance response that facilitates the magnetic tuning reported below.

The permittivity and permeability of the BST and YIG layers, respectively, can be obtained from the effective phase constant using the results from conformal mapping. We generalize the typical expressions ${ }^{11}$ used in the analysis of a CPW line (in which only the permittivity affects the phase velocity of the traveling wave) to include the effect of the permeability

$$
\beta_{\mathrm{eff}}^{2}=\frac{L_{\mathrm{tot}} C_{\mathrm{tot}}}{L_{0} C_{0}}=\frac{\beta_{\mathrm{tot}}^{\prime 2}}{\beta_{0}^{\prime 2}},
$$

where $\beta_{0}^{\prime 2}$ represents the reactance of the CPW line in air

$$
\beta_{0}^{\prime 2}=4 \mu_{0} \varepsilon_{0} \frac{K\left(k^{\prime}\right)}{K(k)},
$$

and $\beta_{\mathrm{tot}}^{\prime 2}$ represents the sum of all of the reactances of the each of the layers

$$
\begin{aligned}
\beta_{t o t}^{\prime 2}= & \sum \beta_{i}^{\prime 2}=\sum 2 \mu_{0} \varepsilon_{0}\left(\mu_{r i} \varepsilon_{r i}\right. \\
& \left.-\mu_{r(i-1)} \varepsilon_{r(i-1)}\right) \frac{K(k)}{K\left(k^{\prime}\right)} \frac{K\left(k_{i}^{\prime}\right)}{K\left(k_{i}\right)} .
\end{aligned}
$$

In these expressions, $K(x)$ is the complete elliptic integral of the first kind with arguments

$$
k=\frac{c}{b} \sqrt{\frac{b^{2}-a^{2}}{c^{2}-a^{2}}}
$$

and

$$
k_{i}=\frac{\sinh \left(\pi c / 2 h_{i}\right)}{\sinh \left(\pi b / 2 h_{i}\right)} \sqrt{\frac{\sinh ^{2}\left(\pi b / 2 h_{i}\right)-\sinh ^{2}\left(\pi a / 2 h_{i}\right)}{\sinh ^{2}\left(\pi c / 2 h_{i}\right)-\sinh ^{2}\left(\pi a / 2 h_{i}\right)}},
$$

where $h_{i}$ is the thickness of the $i$ th layer, $c$ is half the width of the entire CPW line, including the ground planes, $b$ is half the width of the CPW line excluding the ground planes, and $a$ is half the width of the center signal line alone. By using a relative permittivity of 15 and 12 for the YIG layer and the GGG substrate, respectively, we can determine the BST relative permittivity to be $185 \pm 5$ (under zero applied magnetic field, or equivalently, at frequencies above the FMR frequency in the case when the magnetic field is applied). In order to determine the permeability of the YIG, we assume that the BST, YIG, and GGG layers all have a relative permeability equal to 1 in the absence of magnetic fields. Next, under the application of a magnetic field, we see that the effective phase constant changes for frequencies near and below the FMR frequency. Assuming that the permittivity of each layer is unchanged under application of the magnetic field, we can determine the effective squared phase constant of the YIG layer using the above equations. At the low frequency side of FMR, this value for YIG is determined to be $\beta_{\mathrm{eff}}^{2} \sim 105$. Since $\beta_{\mathrm{eff}}^{2}$ represents the product of permittivity and permeability, we conclude that the relative permeability of the YIG at frequencies below FMR is $7 \pm 1$, assuming that the actual relative permittivity of the YIG is 15 . Of course, the permeability increases as the FMR frequency is approached and reduces to 1 at frequencies well above the FMR frequency.

Next, the effective phase constant of the bilayer is tuned under the application of both electric and magnetic fields. Figure 3 shows the effects of dc electric fields, Fig. 3(a), and those of magnetic fields, Fig. 3(b). By keeping the magnetic field constant and increasing the applied electric field [Fig. 3(a)], the effective phase constant shifts toward lower values throughout the frequency band due to the reduction in the permittivity of the BST layer under applied electric field. The permittivity of the BST layer changes from $\sim 185$ to $\sim 154$ as 


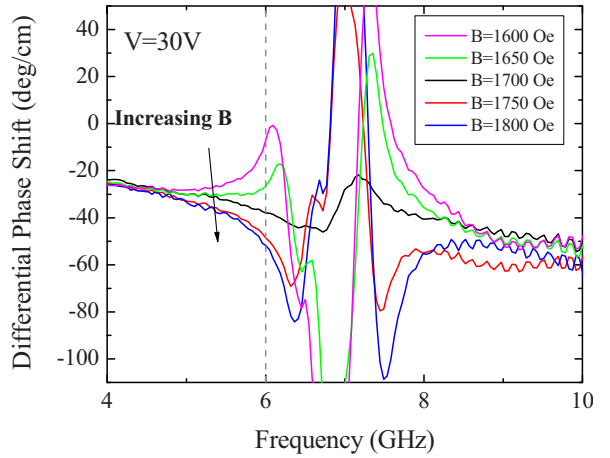

FIG. 4. (Color online) Differential phase shift (degrees/cm) of a $1.1 \mathrm{~mm}$ $\mathrm{CPW}$ line after applying $30 \mathrm{~V}$ to the center line, under various magnetic fields ranging from 1600 to 1800 Oe.

the voltage applied to the lines is increased from 0 to $30 \mathrm{~V}$, representing a tunability of $\sim 16.8 \%$. By keeping the electrical bias at $0 \mathrm{~V}$ and increasing the magnetic field [Fig. 3(b)], the phase profile shifts to higher frequencies without any noticeable change in its shape. At a frequency of $6 \mathrm{GHz}$, the relative permeability of the YIG layer changes from $\sim 18$ to $\sim 12$ for magnetic fields of 1600 Oe and 1800 Oe, respectively. The ability to simultaneously and independently tune the permittivity and permeability of the bilayer structure will allow us to use the CPW line as a phase shifter wherein the impedance $\propto \sqrt{\mu / \varepsilon}$ can be maintained, resulting in lower insertion losses due to improved impedance matching.

We observed no shift in the FMR frequency when the magnetic field was kept constant and the electric field was swept, as shown in Fig. 3(a) and in the inset (expanded view). This fact indicates that the presented structure does not facilitate electric field tuning (through tuning of the permittivity of the BST) of the FMR response. ${ }^{12}$ The main reason for this is that the electric bias voltage was applied across the central signal line and grounds of the CPW structure, and the electric tuning of the dielectric constant occurs only in a small portion of the BST layer which is between the CPW signal and ground lines.

The dielectric and magnetic properties presented in this section demonstrate that these bilayers of YIG/BST are promising for use in tunable phase shifters at microwave frequencies; results from using the structures as phase shifters are presented in Sec. III B.

\section{B. Tunable phase shifters}

The differential phase shift achieved by applying $30 \mathrm{~V}$ to a 1.1-mm-long CPW line is shown in Fig. 4 for magnetic fields ranging from 1600 to 1800 Oe. The differential phase shift is linear in frequency below and above the FMR frequency, as expected for a device with static (in frequency) values of $\mu$ and $\varepsilon$. However, near the FMR frequency, the permeability of the YIG layer is not constant, which results in a deviation from linearity of the differential phase shift curves. Let us consider a narrow band of frequencies just below the FMR frequency around $6 \mathrm{GHz}$. This is the range where the device will be most useful as a tunable phase shifter. As the magnetic field is increased (decreased), the permeability of the YIG at $6 \mathrm{GHz}$ decreases (increases), as

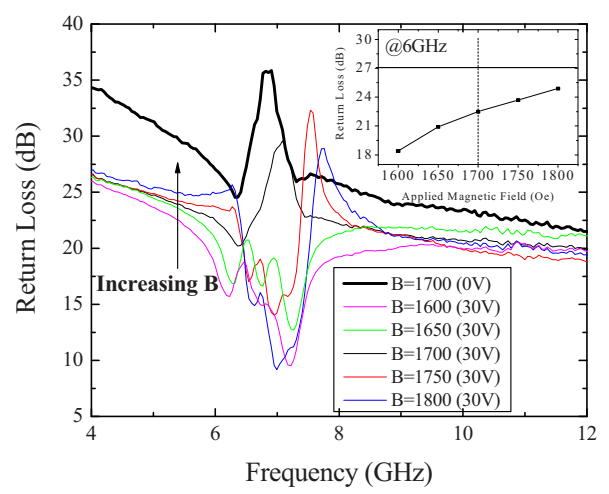

FIG. 5. (Color online) Return loss for the $1.1 \mathrm{~mm}$ CPW phase shifter under zero applied electric field (thick line) and at $30 \mathrm{~V}$ applied electric field under magnetic fields from 1600-1800 Oe. Inset shows the return loss at $6 \mathrm{GHz}$ at $30 \mathrm{~V}$ applied electric field under magnetic fields from 1600-1800 Oe. Horizontal line in inset is the return loss at $0 \mathrm{~V}$ applied field under 1700 Oe (the calibrated condition)

shown in Fig. 3(b). Since the tuning of the phase shifter under applied electric field alone is through a reduction in the permittivity, a reduction (increase) in the permeability would tend to increase (decrease) the overall tuning of the phase constant of the device, as evidenced in Fig. 4. At 6 $\mathrm{GHz}$, the magnitude of the differential phase shift increases from $\sim 38$ to $\sim 52^{\circ} / \mathrm{cm}$ by increasing the magnetic field and decreases to $\sim 8^{\circ} / \mathrm{cm}$ by decreasing the magnetic field. At frequencies very close to FMR $(\sim 6.5-7.5 \mathrm{GHz})$, the loss is exceedingly high, rendering the phase shifter not particularly useful.

In addition to increasing the differential phase shift at frequencies near FMR, the increase in the magnetic field should allow the device to recover the impedance it had before the electric field was applied. Recall that the permittivity decreased after applying an electric field, so if one also decreases the permeability (which is possible at some frequencies by increasing the magnetic field), the loss of the device associated with the mismatch that occurs due to the change in impedance can be eliminated. Such an effect is illustrated in Fig. 5. The heavy line in Fig. 5 shows the return loss of the $1.1 \mathrm{~mm}$ CPW line at zero dc electric field and a magnetic field of $\sim 1700$ Oe. Mismatch occurs throughout the band when the electric field is applied in order to cause a phase shift; this is evidenced by a decrease in the return loss from the value at $0 \mathrm{~V}$. By changing the magnetic field, the return loss changes since the permeability of the YIG changes for frequencies near FMR. At $6 \mathrm{GHz}$, the return loss changes from 27 to $22.4 \mathrm{~dB}$ after application of the electric field. Then, by changing the magnetic field, the return loss can be improved to $24.9 \mathrm{~dB}$ by increasing the magnetic field (inset to Fig. 5). Correspondingly, by decreasing the magnetic field the return loss decreases to $18.4 \mathrm{~dB}$ at $6 \mathrm{GHz}$. Thus, the increase in the applied magnetic field is beneficial in terms of increasing the overall device tuning (phase shift) as well as decreasing the loss associated with impedance mismatch.

After these measurements, the GGG substrate was replaced with a PMN-PT layer to act as a permeability tuner as described in the experiment section above. Because PMN-PT has a high piezoelectric coefficient, by applying an electric field to PMN-PT, we can effectively strain the YIG/BST bi- 


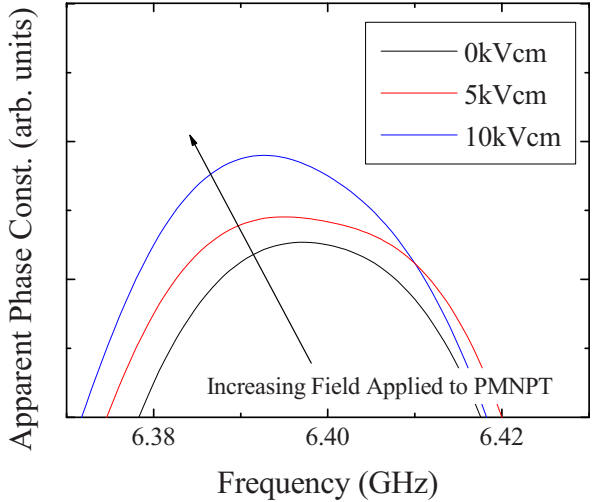

FIG. 6. (Color online) Change in the apparent phase constant as a function of applied field to the PMNPT layer.

layer, causing a shift in the FMR frequency of the YIG through a magnetostrictive effect. ${ }^{7,8}$ This allows for the tuning of permeability without a need to change the magnetic bias field. The motivation for electrical tuning of permeability lies in the fact that the tuning by magnetic field is relatively slow and is costly in terms of power consumption. Figure 6 shows the shift in the phase constant with applied electric field, near the peak in the phase constant, very close to the FMR frequency. By applying a field of only $10 \mathrm{kV} / \mathrm{cm}$, the FMR frequency shifts by $\sim 5 \mathrm{MHz}$. Larger fields could be applied if higher voltages were available or thinner PMN-PT slabs were employed, which would have resulted in a larger shift in the FMR frequency. The exact value of the phase constant is not available due to uncertainty in the calibration stemming from the fact that the not all of the CPW lines survived the polishing/bonding procedure; therefore, only the region of interest near the FMR in Fig. 6 is shown in order to demonstrate the feasibility of using magnetostriction to tune the permeability of YIG. Further work must be done to optimize this procedure.

\section{CONCLUSIONS}

We have reported on the permittivity and permeability tuning of a ferroelectric/ferromagnetic bilayer structure. The structure consists of a BST layer and a YIG layer on a GGG substrate. By applying an electric field, the permittivity of the BST films could be tuned up to $\sim 16.8 \%$ at applied fields of up to $75 \mathrm{kV} / \mathrm{cm}$. Under application of a magnetic field the effect of the varying permeability of the YIG layer was evi- dent. In addition to tuning the phase constant by changing the applied magnetic field through a corresponding change in the permeability of the YIG layer, the YIG layer was used as an impedance matching layer, resulting in an improvement in return loss when the applied magnetic field was increased. Additionally, we demonstrated the feasibility of using a piezoelectric material to mechanically strain the YIG layer, which results in a change in the FMR frequency and subsequently a change in permeability for frequencies near FMR. The additional degree of freedom afforded by these bilayer structures allows phase shifters to be fabricated wherein the impedance mismatch, which arises when the device is tuned, can be effectively eliminated. Such a phenomenon should find particular utility in low loss phased array systems as well as in miniaturized antenna systems.

\section{ACKNOWLEDGMENTS}

The authors would like to thank Dr. W. Chang, Dr. L. M. B. Alldredge, Dr. S. W. Kirchoefer, and Dr. J. M. Pond at the Naval Research Laboratory, as well as Dr. C. Patton at Colorado State University for fruitful discussions. This work is funded by the Office of Naval Research under the directions of Dr. I. Mack and Dr. D. Green.

${ }^{1}$ N. Izyumskaya, Y. I. Alivov, and H. Morkoç, Crit. Rev. Solid State Mater. Sci. 34, 89 (2009).

${ }^{2}$ A. Kozyrev, V. Osadchy, A. Pavlov, and L. Sengupta, IEEE MTT-S Int. Microwave Symp. Dig. 3, 1355 (2000).

${ }^{3}$ W. J. Kim, W. Chang, S. B. Qadri, H. D. Wu, J. M. Pond, S. W. Kirchoefer, H. S. Newman, D. B. Chrisey, and J. S. Horowitz, Appl. Phys. A: Mater. Sci. Process. 71, 7 (2000).

${ }^{4}$ A. B. Ustinov, V. S. Tiberkevich, G. Srinivasan, A. N. Slavin, A. A. Semenov, S. F. Karmanenko, B. A. Kalinikos, J. V. Mantese, and R. Ramer, J. Appl. Phys. 100, 093905 (2006).

${ }_{5}^{5}$ A. B. Ustinov, G. Srinivasan, and B. A. Kalinikos, Appl. Phys. Lett. 90, 031913 (2007).

${ }^{6}$ A. S. Tatarenko, G. Srinivasan, and M. I. Bichurin, Appl. Phys. Lett. 88, 183507 (2006).

${ }^{7}$ S. Shastry, G. Srinivasan, M. I. Bichurin, V. M. Petrov, and S. A. Tatarenko, Phys. Rev. B 70, 064416 (2004).

${ }^{8}$ J. H. Leach, V. Kovalskiy, V. Avrutin, Ü. Özgür, and H. Morkoç, Mater. Res. Soc. Symp. Proc. 1110E, 1110-C06-34 (2008).

${ }^{9}$ R. B. Marks, IEEE Trans. Microwave Theory Tech. 39, 1205 (1991).

${ }^{10}$ J. H. Leach, H. Liu, V. Avrutin, B. Xiao, Ü. Özgür, H. Morkoç, J. Das, Y. Y. Song, and C. E. Patton, J. Appl. Phys. 107, 084511 (2010).

${ }^{11}$ R. N. Simons, Coplanar Waveguide Circuits, Components, and Systems (Wiley-Interscience, New York, 2001).

${ }^{12}$ J. Das, Y.-Y. Song, N. Mo, P. Krivosik, and C. E. Patton, Adv. Mater. (Weinheim, Ger.) 21, 2045 (2009). 